\title{
Measuring Domain Wall Fidelity Lengths Using a Chirality Filter
}

\author{
E. R. Lewis, D. Petit, A.-V. Jausovec, L. O’Brien, D. E. Read, H. T. Zeng, and R. P. Cowburn \\ Department of Physics, Imperial College London, Prince Consort Road, London SW7 2BW, United Kingdom
}

(Received 7 August 2008; published 6 February 2009)

\begin{abstract}
The motion of transverse domain walls (DWs) in thin Permalloy nanowires has been studied by locally detecting the chirality of the moving DW, using a cross-shaped trap acting as a chirality filter. We find that structural changes of the DW occur over a characteristic minimum distance: the "DW fidelity length." The measured field dependence of the fidelity length is in good qualitative agreement with a 1D analytical model and with published results of numerical simulations and experiments. We also demonstrate extension of the fidelity length to meter length scales using a series of filters.
\end{abstract}

DOI: 10.1103/PhysRevLett.102.057209

PACS numbers: 75.75.+a, 75.60.Ch, 75.60.Jk, 85.70.Kh

The controllable motion of magnetic domain walls (DWs) in nanoscale conduits is fundamental to the operation of new magnetic logic and data storage devices $[1,2]$. In particular, changes of the DW structure are significant in device applications where DW motion is controlled via interaction with artificial defects, because the detailed spin distribution in the wall affects the nature and strength of the pinning potential [3]; the fidelity of data transmission may then depend on preserving the DW structure. Simulations predict that for thin and narrow nanowires, the equilibrium wall structure is a transverse DW $[4,5]$ characterized by a charge (head to head or tail to tail) and a chirality (defined by the sense of rotation of spins in the wall). The dynamics of a single DW can be qualitatively described using analytical models [6-8], which predict two regimes of motion: viscous below a critical "Walker field" and oscillatory above it. In the particular case of a transverse DW in a nanostrip, full micromagnetic simulations are necessary to elucidate the details of the wall motion and show that the oscillation of the DW structure proceeds via successive nucleation and annihilation of antivortices, leading to periodic transformations between transverse DWs of opposite chirality. For device applications it is necessary to know the length scale over which the chirality is predictable in a real system. Experimentally, such behavior is difficult to measure because the evolution of the DW structure occurs on nanosecond time scales and the absolute moment of the DW is small (on the order of $10^{-13} \mathrm{emu}$ ); however, temporal oscillations of the nanowire resistance (due to the anisotropic magnetoresistance effect) have been observed, corresponding to periodic transformations of the DW structure [9], and the DW velocity oscillation which accompanies the structural transformations has been detected using time-resolved magneto-optical Kerr effect (MOKE) measurements [10]. In this Letter, we demonstrate direct spatial measurements of the position dependence of the DW structure as it propagates along such wires. We construct a "chirality filter" consisting of a cross-shaped trap, which allows us not only to detect the DW chirality but also to reset it. We find that transformations of the DW structure occur over a characteristic distance which we call the "DW fidelity length"; the measured field dependence of the fidelity length is in good qualitative agreement with a $1 \mathrm{D}$ analytical model of DW motion, numerical simulations, and published experimental results. In addition, we exploit the resetting property of the filter to demonstrate extension of the fidelity length to meter length scales using a series of crosses.

Permalloy nanowires $90 \mathrm{~nm}$ wide and $12 \mathrm{~nm}$ thick (Fig. 1) were fabricated on Si substrates using electron beam lithography, thermal evaporation, and a lift-off process, and characterized using spatially resolved longitudinal MOKE measurements. The focused laser spot has a diameter of $\sim 5 \mu \mathrm{m}$ and is positioned on the far right-hand part of the structure; typical MOKE loops for each of the measured switching patterns are shown in Fig. 2(b). The first half of the applied field sequence and the magnetization configurations obtained in the absence of chirality

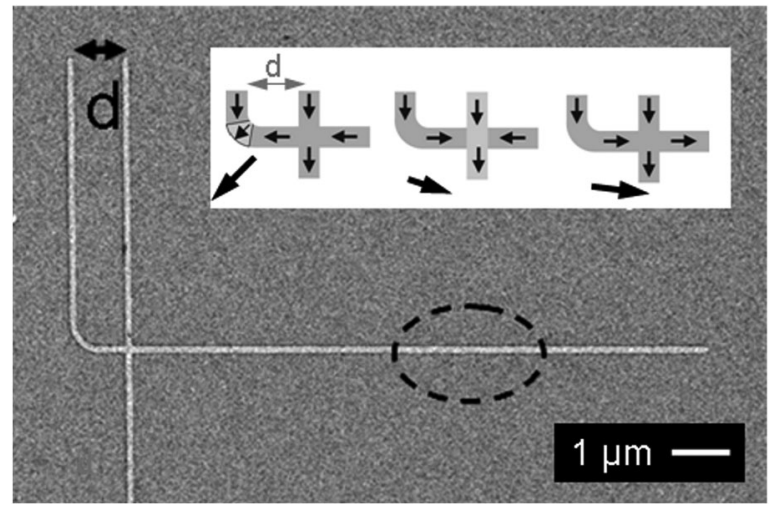

FIG. 1. Scanning electron microscopy (SEM) image of an $L$-shaped nanostructure with a cross at a distance $d$ from the corner (the lower part of the transverse arm extends $5 \mu \mathrm{m}$ below the cross); the position of the MOKE measurement is indicated by the dashed ellipse. Inset, left to right: First half of field sequence (black arrows) and schematics indicating magnetization configurations in the absence of chirality reversal. 


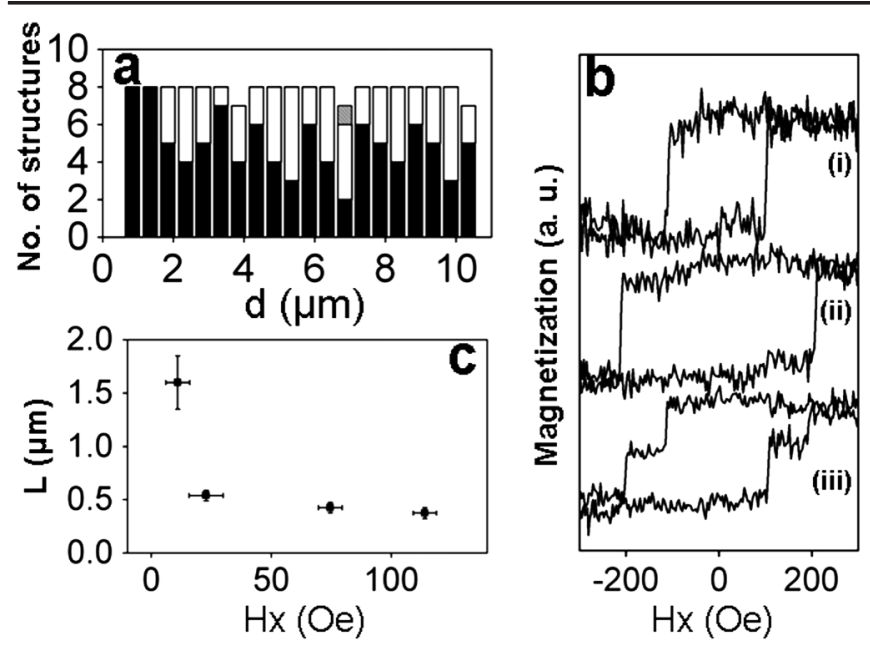

FIG. 2. (a) Number of structures showing each type of switching pattern as a function of distance $d$. Black: Switching corresponding to the DW reaching the cross with the chirality set by the initial field pulse; gray: switching corresponding to DW reaching the cross with the opposite chirality; white: double transition. Chirality reversal is not observed for $d \leq 1.35 \mu \mathrm{m}$. (b) Typical MOKE loops, vertically offset for clarity. (i), (ii) MOKE loops showing single transitions at $H_{T}$ and $H_{N}$. (iii) MOKE loop showing double transition. (c) Measured fidelity length $L$ as a function of field.

reversal are shown schematically in the insets of Fig. 1; the second half of the sequence is exactly equivalent to the first, with all fields reversed. The left-hand end of each structure is patterned into an $L$ shape, and a transverse arm crossing the main wire is patterned at a distance $d$ from the corner. A DW is created in one corner by application of a magnetic field of magnitude $\sim 600$ Oe oriented at $45^{\circ}$; the magnetization in the transverse arm is also reset at this stage, so that the DW is initially created with core magnetization parallel to the magnetization in the transverse arm. Once the DW is created, a horizontal field is applied to move it along the wire. (Note that the maximum rate of change of the field is $\sim 4 \mathrm{kOe} / \mathrm{s}$ : it is quasistatic.) The field required to move the DW out of the corner is the "propagation field" $H_{P}$; measurements on 15 structures give $H_{P}=11 \pm 5$ Oe. The DW chirality is detected using the "chirality filtering" property of the transverse arm: the magnetization in this arm is constrained to lie in the transverse direction by the shape anisotropy of the wire, and transverse DWs approaching the filter therefore have core magnetization either parallel or antiparallel to the magnetization in the transverse arm. In the parallel case, it is energetically favorable for the DW to sit inside the cross, so that the DW is pinned there until the "transmission field" $H_{T}$ is applied. In the antiparallel case, the DW is blocked just in front of the cross, and switching of the right-hand part of the structure only occurs via nucleation of a new domain at the cross, at the "nucleation field" $H_{N}$. (MOKE measurements to the left of the cross confirm that a DW does travel to the cross: the magnetization in this region switches at $\pm H_{P}$.) We measure $H_{T}=115 \pm 10 \mathrm{Oe}$ and $H_{N}=210 \pm 10 \mathrm{Oe}$; the large separation of the fields allows the parallel and antiparallel cases to be distinguished unambiguously.

By varying the distance $d$ (see Fig. 1), we can examine how the DW structure changes as it propagates along the wire; $d$ was increased in $500 \mathrm{~nm}$ increments, starting from $850 \mathrm{~nm}$. Each measurement was averaged over 600 cycles of the applied field. We observe three different switching patterns [see Fig. 2(b)]: a single transition at the field corresponding to the DW reaching the cross with the chirality set by the initial field pulse (switching at $115 \mathrm{Oe}$ ), a single transition corresponding to the opposite chirality (switching at $210 \mathrm{Oe}$ ), and a mixed situation where both transitions are seen. (The origin of the double transitions is assumed to be stochastic DW motion as described in the text below and in Ref. [11].) The number of structures showing each type of switching as a function of $d$ is shown in Fig. 2(a). We first note that the data separate into two regimes: at small values of $d \leq$ $1.35 \mu \mathrm{m}$, only one transition is seen for each of the 16 structures measured, but at larger values of $d \geq 1.85 \mu \mathrm{m}$ either a single or a double transition [Fig. 2(b) (iii)] may be observed. The double transitions are attributed to the onset of chirality reversal, part of the characteristic behavior of Walker breakdown. However, our data do not provide evidence of periodic behavior: neither the incidence of double transitions nor the probability of reversal extracted from the relative heights of the two steps in the double transitions are periodic. Instead, we can fully predict the DW chirality only at distances up to $1.6 \pm 0.25 \mu \mathrm{m}$; the DW chirality is always preserved over this distance, leading to the definition of a phenomenological "DW fidelity length" $L . L$ is determined from the experimental data as the maximum distance for which the DW structure is always preserved when starting from a known initial state; equivalently, it is the distance at which double transitions first appear. Note that because $L$ is associated with the spatial half-period of the predicted oscillations and $L=$ $1.6 \pm 0.25 \mu \mathrm{m}$, the full spatial period is $\sim 2-3 \mu \mathrm{m}$, so we expect that our spatial sampling period of $500 \mathrm{~nm}$ should be sufficient to see the periodicity if it is present.

The lack of periodicity can be attributed to two sources of disorder: structural defects and thermal effects. Numerical simulations have shown that edge roughness can increase $H_{W}$ or even suppress Walker breakdown entirely [12]. Variation in edge roughness along a particular structure and between structures could therefore lead to local suppression of breakdown, or pin the DW so that its subsequent motion along a smoother section of wire occurs above the Walker field. Although such effects can explain the lack of periodicity in the data, it is more difficult to see how they account for the measured double transitions. The double transitions are consistent with stochastic DW be- 
havior, where application of the same sequence of fields can produce different DW dynamics [11]. We attribute this to thermal effects. Numerical simulations including the effect of temperature [13] show that for fields smaller than the zero-temperature depinning field, the depinning is thermally activated and takes place at a distribution of fields which may extend both above and below $H_{W}$. The simulated zero-temperature depinning field for edge roughness with typical Permalloy grain size $10 \mathrm{~nm}$ is $\sim 20$ Oe [13]; if the spatial extent of the pinning potential is assumed to be comparable to the grain size, the corresponding energy barrier is on the order of $\sim 5 k_{B} T$ at $300 \mathrm{~K}$ and the characteristic "wait time" for thermal depinning (in zero field) $\tau=\tau_{0} \exp \left(E_{\text {barrier }} / k_{B} T\right)$ is $15 \mathrm{~ns}$, where the attempt frequency $1 / \tau_{0}$ is typically $10^{10} \mathrm{~Hz}$ [14]. Our maximum field sweep rate is $\sim 4 \mathrm{kOe} / \mathrm{s}$, corresponding to a 20 Oe increase over $5 \mathrm{~ms}$, so it is expected that thermally activated processes are important.

Although the DW dynamics are complex and include some retrograde motion, as a first approximation we expect that $L$ will have the same dependence on field as the average spatial half-period of the predicted transformations of the wall structure. The period $T$ of the oscillations is given by [8]

$$
T=\frac{2 \pi\left(1+\alpha^{2}\right)}{\gamma_{0} H_{W} \sqrt{\left(H_{a} / H_{W}\right)^{2}-1}},
$$

where $H_{a}$ is the applied field, $H_{W}$ is the Walker field, $\alpha$ is the damping constant, and $\gamma_{0}=\mu_{0} \gamma$ where $\gamma$ is the electron gyromagnetic ratio. Good agreement with this expression has been obtained experimentally [9]. The spatial halfperiod, $L_{0}$, is given by $L_{0}=v_{\mathrm{av}} T / 2$, where $v_{\mathrm{av}}$ is the average DW velocity. Experimentally, the average velocity initially decreases with field above $H_{W}$, passes through a minimum, and increases linearly with field at high fields $[9,15]$, in qualitative agreement with theory and simulations $[8,10,16,17] . L$ is therefore expected to decrease with field and tend to a constant at high fields. By decreasing the radius of curvature $R$ in the corner where the DW is created, we are able to increase the field at which the DW depins and moves towards the cross; $R$ is measured from the center of the wire. The measured $L$ for four different depinning fields is shown in Fig. 2(c): the first point corresponds to the data of Fig. 2(a), where $R=$ $450 \mathrm{~nm}$, the second and third to depinning from corners with $R=200$ and $50 \mathrm{~nm}$, respectively, and the fourth to depinning from a cross. (The spatial sampling period for these three higher fields was $50 \mathrm{~nm}$.) The expected decrease of $L$ with field is seen, with $L$ tending to around $350 \mathrm{~nm}$ at high fields. For the three higher fields, we can test our association of the fidelity length $L$ with the spatial half-period $L_{0}$ of the predicted oscillations by estimating an average velocity using Eq. (1) and $v_{\text {av }}=2 L / T$, where we have replaced the spatial half-period $L_{0}$ by the fidelity length $L$. (A meaningful calculation is not possible for the lowest field because $T$ diverges close to $H_{a}=H_{W}$ and the value of $H_{W}$ is not accurately known.) $H_{W}$ has been measured on a $200 \mathrm{~nm} \times 10 \mathrm{~nm}$ Permalloy strip as $\sim 14$ Oe [9]; we assume that for our wires, $H_{W}$ lies within the range of propagation fields. Taking $H_{W}=H_{P}=11 \pm$ 5 Oe, for depinning fields of 23,75 , and 115 Oe we obtain $v_{\text {av }}=61 \pm 9,177 \pm 3,240 \pm 1 \mathrm{~m} / \mathrm{s}$, well within the range of published experimental measurements $[9,15,18]$; allowing $H_{W}$ to vary between 6 and 16 Oe gives a change of no more than $20 \%$ in these values. Note also that we have neglected any influence of the curvature of the corner on the DW chirality. However, such effects would only be important on the curved section of the nanowire, which is only $40 \mathrm{~nm}$ for $R=50 \mathrm{~nm}$, and measurements of the depinning field as a function of $R$ show that for $R=$ $450 \mathrm{~nm}$ there is no intrinsic pinning due to the curvature. For $R=200 \mathrm{~nm}$ there is some pinning due to the curvature, but the effect is small (the increase in pinning field is only $\sim 12 \mathrm{Oe}$ ). We therefore argue that curvature effects are insignificant because they are either intrinsically small or only important over small distances. One might additionally consider the fact that on the curved section of the wire the component of the field parallel to the wire axis is reduced. However, the resulting maximum variation in the field is $30 \%$, dropping to $8 \%$ when the DW has moved halfway along the curved section; the spreads in propagation field values on nominally identical structures for $R=$ $450 \mathrm{~nm}$ and $R=200 \mathrm{~nm}$ are comparable or larger (45\% and $30 \%$, respectively).

We have also used a series of chirality filters to artificially extend $L$. We fabricated two types of "comb" nanostructure, consisting of an $L$-shaped or $U$-shaped nanowire
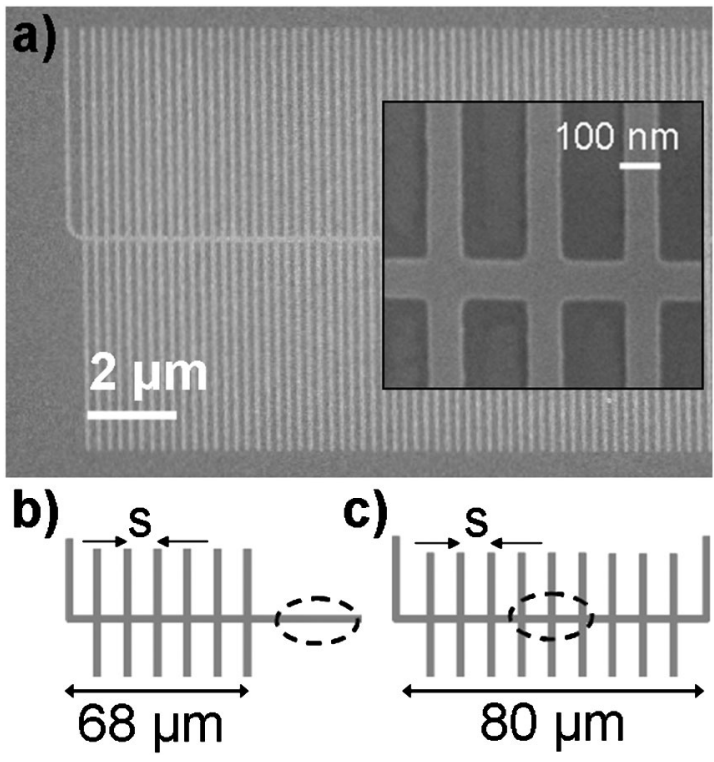

FIG. 3. Comb structures. (a) SEM image of left-hand part of comb structure with cross spacing $s=250 \mathrm{~nm}$. (b) Schematic of $L$-shaped comb. (c) Schematic of $U$-shaped comb. The position of the MOKE measurement is indicated by the dashed ellipse. 
with many transverse arms of center-to-center spacing $s$ crossing the main wire (Fig. 3). The measurement on the $L$ shapes is the same as in the single filter case (cf. Fig. 1); the switching field is measured on the far right-hand side of the structure. Most of the DW motion takes place at $H_{T}=$ $115 \mathrm{Oe}$, and at this field $L$ has been measured as $375 \pm$ $25 \mathrm{~nm}$ [last point of Fig. 2(c)]: for $s \geq 400 \mathrm{~nm}$, double transitions [cf. Fig. 2(b) (iii)] are observed, but when $s \leq$ $350 \mathrm{~nm}$, only the lower-field $\left(H_{T}\right)$ transition is seen. Because the latter case corresponds to preservation of the wall chirality throughout the filter array, which extends over $68 \mu \mathrm{m}, L$ has effectively been increased to $>68 \mu \mathrm{m}$. To determine the limit of the extension of $L$, we fabricated $U$-shaped nanowires with transverse arms of spacing $s=$ $250 \mathrm{~nm}$ (Fig. 3). A DW was created in one corner by application of a large field pulse, followed by an oscillating horizontal field with maximum magnitude in between $H_{T}$ and $H_{N}$, so that a DW in the parallel configuration could transmit, but no new domains could be nucleated. In the absence of chirality reversal, the DW will move back and forth between the corners, but if the DW chirality reverses at any point, it will not be able to transmit through the crosses and the magnetization switching at the center of the structure will stop. After 40000 cycles of the horizontal field, we still measured switching, corresponding to chirality-conserving motion of the DW through more than $25 \times 10^{6}$ crosses, over a total distance of $3.2 \mathrm{~m}$. Simulations of the magnetization configuration in the cross show that the transverse component of the magnetization falls to $10 \%$ of its maximum value at a distance of $50 \mathrm{~nm}$ either side of the cross. We therefore expect that if the DW magnetization distribution does not overlap this $50 \mathrm{~nm}$ region, the DW motion will be largely unaffected by the presence of the cross. Within this region, the motion is perturbed by exchange interactions with the spins in and around the cross, which favor parallel alignment; as the DW passes through a series of crosses, its chirality is periodically "reset."

In conclusion, we have characterized DW motion in Permalloy nanostrips by detecting the chirality at specific points in the DW trajectory using a cross-shaped trap acting as a chirality filter. We do not measure spatially periodic behavior, but instead find that transformations of the wall structure are stochastic and occur over a characteristic minimum distance: the "DW fidelity length." We have measured the field dependence of the fidelity length and find that it decreases with driving field (as predicted by the
1D model, simulations, and experiments): at low fields close to the intrinsic propagation field of the wire, the fidelity length is $1.6 \mu \mathrm{m}$, decreasing asymptotically to $350 \mathrm{~nm}$ at high fields. Finally, by using a series of crosses, we have succeeded in extending the effective fidelity length to meter length scales: the DW chirality can be maintained in this way over arbitrarily long distances, which is crucial for chirality-dependent DW devices.

[1] D. A. Allwood et al., Science 309, 1688 (2005).

[2] S. S. P. Parkin, M. Hayashi, and L. Thomas, Science 320, 190 (2008).

[3] D. Petit et al., J. Appl. Phys. 103, 114307 (2008).

[4] Y. Nakatani, A. Thiaville, and J. Miltat, J. Magn. Magn. Mater. 290-291, 750 (2005).

[5] R. D. McMichael and M. J. Donahue, IEEE Trans. Magn. 33, 4167 (1997).

[6] N. L. Schryer and L. R. Walker, J. Appl. Phys. 45, 5406 (1974).

[7] M. A. Slonczewski, Magnetic Domain Walls in Bubble Materials (Academic, New York, 1979).

[8] A. Thiaville and Y. Nakatani, Spin Dynamics in Confined Magnetic Structures (Springer, Berlin, 2006), Vol. III, p. 161.

[9] M. Hayashi et al., Nature Phys. 3, 21 (2007).

[10] J. Yang et al., Phys. Rev. B 77, 014413 (2008).

[11] Double transitions could be caused by the DW stopping partway through the MOKE spot due to pinning by a defect, with the higher switching field corresponding to depinning from the defect (or renucleation next to the defect). However, the measured higher switching field is always $H_{N}$, which could only be caused by a large defect such as the cross or a break in the wire. SEM imaging of the structures showed that such large defects were never accidentally formed, and that breaks in the structures were very rare. We therefore ascribe the double transitions not to this mechanism but to stochastic DW behavior as described in the text.

[12] Y. Nakatani, A. Thiaville, and J. Miltat, Nature Mater. 2, 521 (2003).

[13] E. Martinez et al., Phys. Rev. B 75, 174409 (2007).

[14] G. Bertotti, Hysteresis in Magnetism (Academic, New York, 1998).

[15] G. S. D. Beach et al., Nature Mater. 4, 741 (2005).

[16] J. Y. Lee et al., Phys. Rev. B 76, 184408 (2007).

[17] S. K. Kim et al., arXiv:0804.2301v1.

[18] D. Atkinson et al., Nature Mater. 2, 85 (2003). 